\title{
ON $k$-ANALOGUES OF DIGAMMA AND POLYGAMMA FUNCTIONS
}

\author{
EMRAH YILDIRIM AND İNCI EGE
}

Abstract. In this work, we obtain some integral representations of $k$-analogue of classical digamma function $\psi(x)$. Then by using the concepts of neutrix and neutrix limit, we generalize the $k$ digamma function $\psi_{k}(x)$ and the $k$-polygamma function $\psi_{k}^{(r)}(x)$ for all real values of $x, r \in \mathbb{N}$ and $k>0$. Also further results are given.

Mathematics subject classification (2010): 33B15, 33B99.

Keywords and phrases: Digamma function, polygamma function, $k$-digamma function, $k$-polygamma function, neutrix.

\section{REFERENCES}

[1] M. Abramowitz And I. A. Stegun (Eds), Handbook of Mathematical Functions with Formulas, Graphs, and Mathematical Tables, National Bureau of Standards, Applied Mathematics Series 55, Washington, 1970.

[2] J. G. VAN DER CORPUT, Introduction to the neutrix calculus, Journal d'Analyse Mathématique 7, 1 (1959), 281-398.

[3] R. Diaz and E. Pariguan, On hypergeometric functions and Pochhammer k-symbol, Divulgaciones Matemáticas 15, 2 (2007), 179-192.

[4] İ. Ege And E. Yildirim, Some generalized equalities for the q-gamma function, Filomat 26, 6 (2012), 1227-1232.

[5] B. Fisher And Y. Kuribayashi, Neutrices and the gamma function, J. Fac. Ed. Tottori Univ. Math. Sci. 36, 1-2 (1987), 1-7.

[6] B. Fisher And Y. Kuribayashi, Some results on the gamma function, The Journal of Faculty of Education Tottori University 37, 2 (1988).

[7] B. Fisher And A. Kiliçman, Some results on the gamma function for negative integers, Appl. Math. Inform. Sci. 6, 2 (2012), 173-176.

[8] I. S. GRAdShteyn AND I. M. RYZhik, Table of integrals, series, and products, Academic press, 2014.

[9] B. Jolevska-Tuneska And I. Jolevski, Some results on the digamma function, Appl. Math. Inform. Sci. 7, 1 (2013), 167-170.

[10] V. KRASNIQI, Inequalities and monotonicity for the ration of $k$-gamma function, Scientia Magna 6, 1 (2010), 40-45.

[11] C. G. Kokologiannaki, Properties and inequalities of generalized k-gamma, beta and zeta functions, Int. J. Contemp. Math. Sciences 5, 14 (2010), 653-660.

[12] C. G. Kokologiannaki And V. KRASniQI, Some properties of the k-gamma function, Le Matematiche 68, 1 (2013), 13-22.

[13] M. Mansour, Determining the $k$-generalized gamma function $\Gamma_{k}(x)$ by functional equations, Int. J. Contemp. Math. Sciences 4, 21 (2009), 1037-1042.

[14] E. ÖZÇAG̃, Results on compositions involving Dirac-delta function, In AIP Conference Proceedings 1895, 1 (2017), 050007.

[15] A. Rehman, S. Mubeen, N. SAdiq And F. Shaheen, Some inequalities involving k-gamma and $k$-beta functions with applications, Journal of Inequalities and Applications 2014, 1 (2014), 224.

[16] A. SALEM AND A. KILIÇMAN, Estimating the polygamma functions for negative integers, Journal of Inequalities and applications 2013, 1 (2013), 523. 
[17] A. SAlEm, The neutrix limit of the q-Gamma function and its derivatives, Applied Mathematics Letters 23, 10 (2010), 1262-1268.

[18] A. SALEM, Generalized the q-Digamma and the q-Polygamma Functions via Neutrices, Filomat 31, 5 (2017), 1475-1481.

[19] Z. Sun, A. Li AND H. QIN, The Neutrix Limit of the Hurwitz Zeta Function and Its Application, IAENG Int. J. Appl. Math. 47, 1 (2017), 56-65.

[20] J. Zhang AND H. N. SHI, Two double inequalities for k-gamma and k-Riemann zeta functions, Journal of Inequalities and Applications 2014, 1 (2014), 191. 